\title{
A productivity prediction method for condensate gas reservoir
}

\author{
Huang QuanHua ${ }^{1}$, Ding HongJun ${ }^{1}$, Lin XingYu*1 \\ ${ }^{1}$ Petroleum engineering school, Southwest petroleum university, Chengdu, Sichuan, 610500, china
}

\begin{abstract}
At present, multiphase flow productivity calculation requires many parameters, and most of them only consider oil and gas two-phase flow, which is complicated and limited. Therefore, a reasonable productivity formula of condensate gas reservoir with producing water is needed. The three-zone model of condensate gas reservoirs is generally applied to the physical model for inferring productivity. On this basis, an improved model is established, which includes that different seepage characteristics are considered for different zones. Moreover, the effects of inclined angle and water production on gas wells are regarded as pseudo-skin factors and additional-skin factors. In addition, Zone I considers the effects of high-speed nonDarcy effect(HSND), starting pressure gradient, stress sensitivity, inclined angle and water production; Zone II is the same way excepting starting pressure gradient and stress sensitivity; Zone III only considers the effects of inclined angle and water production. As a result, a productivity equation with multiple factors for condensate gas wells is established. Through analysing cases and influences in $\mathrm{H}$ gas reservoir X1 well, the HSND, starting pressure gradient, stress sensitivity and water production have a negative impact on gas well productivity, but the inclined angle is opposite. Founded that the starting pressure gradient impacts on productivity is less than the HSND because of the limited radius of Zone I; the impact of the HSND on productivity increases with the decreasing of bottom hole pressure; the impact of water production on gas well productivity is much higher. When the angle is over $60^{\circ}$, the effect of gas
\end{abstract}

\section{Introduction}

At present, there are two ideas for analyzing deliverability test of condensate gas wells: Converting the production of condensate oil production into condensate gas, that is, oil and gas two-phase fluids are regarded as pseudo-singlephase fluid for productivity evaluation. This method is suitable for high gas-liquid ratio. For the underground gasliquid two-phase seepage, the pseudo-pressure method is used to evaluate the productivity. The process of calculating the pseudo-pressure is relatively complicated, and factors such as the property of the gas-liquid fluid, the phase state and the oil-gas relative permeability need to be considered. In the case of low gas-liquid ratio, the calculation result is more reliable.

Due to the high gas-liquid ratio in $\mathrm{H}$ gas reservoirs, this paper treats the two phases of oil and gas as one phase. Firstly, we converted the production of formation condensate oil to the condensate gas for calculation. Secondly, we simplified the three-phase problem to the two-phase problem (gas and water). Thirdly, we established physical models and mathematical models by considering different influences in different zones after considering the influence of water production on gas wells as additional-skin factor. Eventually, we determined the gas well productivity equation.

Fevang and Whitson[1]divided the condensate gas reservoir into three seepage zones with different characteristics; Gringarten[2] used the method of well test interpretation to subdivide the seepage zone on the basis of three into four. The productivity of condensate gas reservoirs can be solved and the boundary can be determined by numerical simulation. However, in order to solve the productivity theoretically, zone is generally divided first. And then, we can get the productivity equation. For low-permeability gas reservoirs, it is often effected by starting pressure gradient and slippage effect; when the flow rate is faster, HSND will happen in the gas reservoir. Lu and Jalal Mazloom et al. [4-5] evaluated the productivity of condensate gas wells by using the twophase pseudo-pressure method. Johnson[6] used production data to calculate the gas well productivity by using the pseudo-single-phase pressure method. Zhixiong $\mathrm{He}$ and Quanhua Huang[7-9] also evaluated the productivity of condensate gas wells.

Water breakthrough in gas reservoirs has a greater impact on productivity with water lock effect in usual, which greatly reduces the productivity of gas wells. In engineering, pseudo-skin factor is often used to describe the impact of inclined well on productivity. Since the

\footnotetext{
* Corresponding author: cnlinxingyu@163.com
} 
larger the value of $\theta$ is, the greater the communication area between the well bore and the formation will be. Therefore, the greater the inclined angle is, the greater the productivity will be.

\section{Model building}

Since $\mathrm{H}$ gas reservoir was producing with water, based on the actual situation, a three-zone three-phase seepage

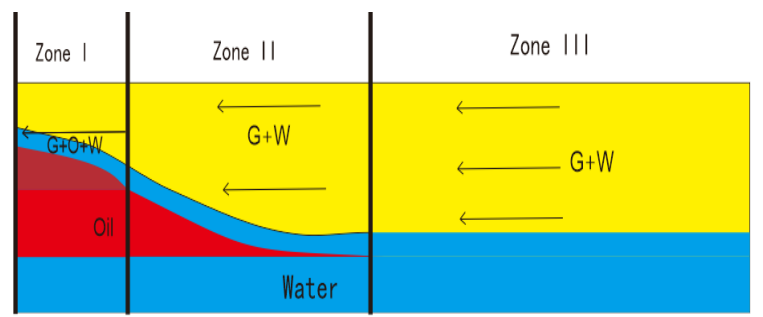

Figure 1. Schematic diagram

As shown in Figure 2, one micro-element is taken from each of the three zones. Zone I is a three-phase flow of oil, gas and water. Considering condensate oil saturation and water saturation, the irreducible water saturation is the original irreducible water saturation. The condensate saturation is the sum of the critical condensate flow saturation and the flow condensate saturation; the condensate saturation of Zone II does not reach the critical flow saturation, and the irreducible water saturation is the original irreducible water saturation; there is no condensate oil in Zone III, and the irreducible water saturation is the original irreducible water saturation. Assuming that the water saturation in the three zones remains unchanged, then the gas saturation will continually decrease.

\section{Productivity formula}

According to the physical model of each zone, combined with the seepage characteristics, different influencing factors are considered in different zones. For condensate gas reservoirs, depletion mining is often used for production. As the bottom hole pressure decreases, the model expands from Zone III to Zone I, and the fluid seepage characteristics change constantly. From Darcy seepage to non-Darcy seepage in part of zones, stress sensitivity and starting pressure gradient are considered in part of zones too. Therefore, this paper derives the productivity equation under different bottom hole pressure.

Due to the influence of inclined and gas reservoirs with producing water, we can obtain the productivity formula of low-permeability, inclined, water-producing condensate gas reservoirs under these conditions in three zones: model as shown in Figure 1 is established. The model assumptions are as follows:(1) The condensate gas reservoir is a homogeneous, isothermal, equal thickness cylindrical gas reservoir;(2) The porosity and permeability of the gas reservoir do not change with pressure and temperature, it also leave out the stress sensitivity effect;(3) Ignoring absorptive effect and diffusion effect;(4) Ignoring the inertia force and gravity;(5) Ignoring condensate water.

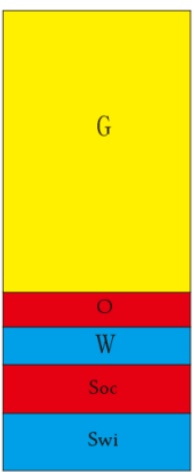

Zone I

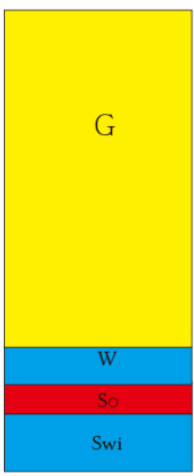

Zone II

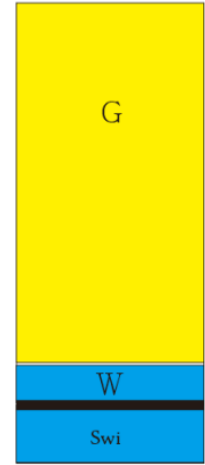

Zone III
Figure 2. Schematic diagram of fluids

$$
\begin{gathered}
\int_{p_{w i}}^{p_{r r}} \frac{e^{-\alpha\left(p_{i}-p\right)}}{\mu Z} 2 p \mathrm{~d} p+\int_{p_{o r}}^{p_{o}} \frac{2 p}{\mu Z} \mathrm{~d} p-\lambda \int_{r_{r o}}^{r_{c r}} \frac{e^{-\alpha\left(p_{i}-p\right)}}{\mu Z} 2 p \mathrm{~d} r=\frac{q_{s c}}{\pi h k k_{r g}} \frac{p_{s c} T}{T_{s c}} \ln \left(\frac{r_{e}}{r_{w o}}+S_{\theta}+S_{b}\right)+ \\
+\beta \rho_{s c} \frac{1}{2 \pi^{2} h^{2} \mu} \frac{p_{s c} T}{T_{s c}}\left(\int_{r_{w o}}^{r_{c c}} \frac{e^{-\alpha\left(p_{i}-p\right)}}{r^{2}} d r+\frac{1}{r_{c r}}-\frac{1}{r_{d}}\right) q_{s c}{ }^{2}
\end{gathered}
$$

We determine the productivity equation when the gas well breaks through water by modifying the skin coefficient, called $\mathrm{S}$ :

$$
S_{b}=\left(\frac{1}{K_{r g}}-1\right) \ln \left(\frac{r_{b}}{r_{w f}}\right)
$$

\section{Example calculation}

It is known that the thickness of the gas well $\mathrm{X} 1$ in the $\mathrm{H}$ gas reservoir is $12 \mathrm{~m}$, the seepage is $0.5 \mathrm{mD}$, the relative permeability of the gas phase is 0.6 under the gas-water two-phase seepage condition, the gas reservoir temperature is $348 \mathrm{~K}$, the dip angle between the pipe string and the vertical plane is $70^{\circ}$, the original formation pressure is $30 \mathrm{MPa}$ and the bottom hole radius is $0.1 \mathrm{~m}$. After the well has been in production for a period of time, condensate oil was precipitated, at the same time, the well has been producing water continuously.

We can determine the dew point pressure through the phase diagram. Regardless of the presence of condensate water in the gas phase, a schematic diagram of the phase state is obtained through software fitting and experiments. The average formation pressure of $\mathrm{H}$ gas reservoir is $32 \mathrm{Mpa}$, and the average formation temperature is $350 \mathrm{~K}$. The experiment shows that the dew point pressure at this temperature is $26 \mathrm{MPa}$ with a lower producing pressure difference, which makes it easy to precipitate condensate oil in the formation.

The CVD experiment is to simulate the production performance during the depletion development process of 
the condensate gas reservoir, whose purpose of predicting the change of the formation retrograde condensate oil saturation during this process. And we can determine the recovery percent of the natural gas and condensate oil at each grade of pressures.

Since adopting the method of converting the three phases of oil, gas and water into gas is used to predict the productivity, the schematic diagram of the viscosity relationship calculated by Lee's method is as the figure 3 .

We can determine the radius under different pressures through the relationship between the formation pressure curve and the radius. To adopt pseudo-pressure to

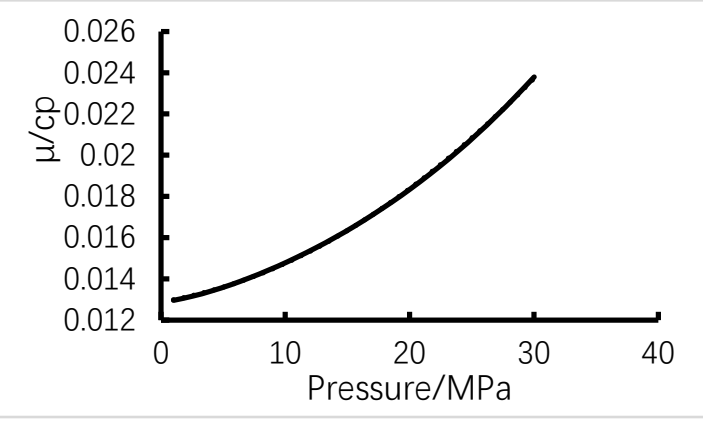

Figure 3. Viscosity change of condensate gas represent pressure changes in different zones, we can get the value of stress sensitivity coefficient in Zone I is 0.001 . The rectangular approximate integration method is used to calculate the pseudo pressure under different bottom hole pressures.

The figure below is a schematic diagram of the radius of Zone I and Zone II corresponding to different bottom hole pressures. From the figure 5, it can be seen that condensate oil begins to deposit when the pressure is lower than the dew point pressure. And it only start to flow after reaching certain conditions to form Zone I.

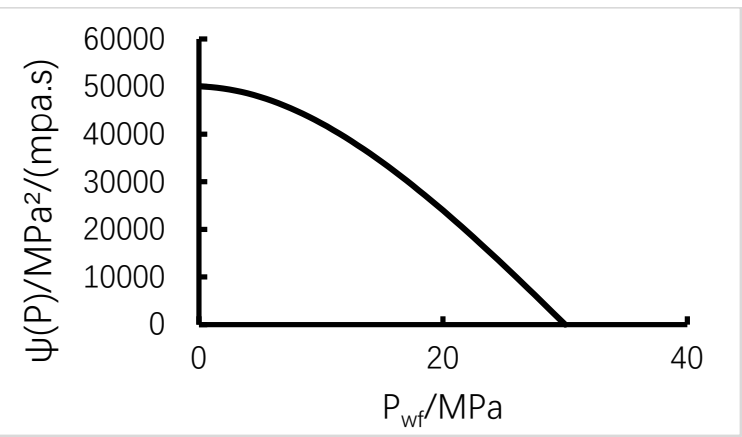

Figure 4. Pseudo pressure change graph

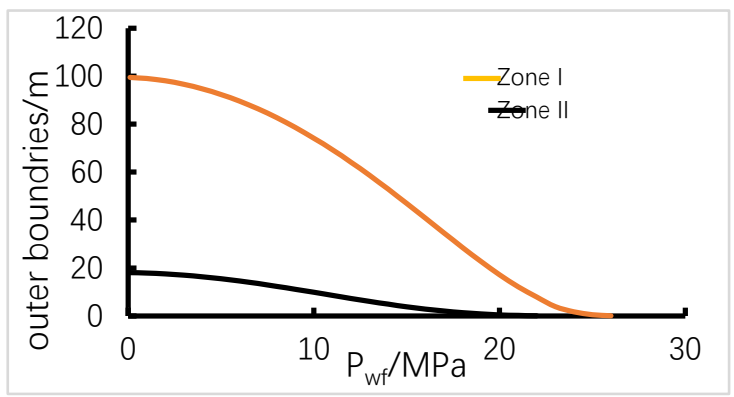

Figure 5. Changes in the outer boundaries

Productivity calculation. In this section, the productivity of gas well $\mathrm{X} 1$ in the $\mathrm{H}$ gas reservoir is calculated by combining experimental and theoretical methods, and correspondence between different bottom hole pressure and daily gas production are calculated separately within the three pressure ranges. Since the method used in different pressure ranges corresponds to different seepage characteristics, the model used in this paper can be more practical, in which the stress sensitivity coefficient is 0.001 and the starting pressure gradient is $0.01 \mathrm{MPa} / \mathrm{m}$.

\section{Analysis of influencing factors}

It can be seen from Figure 8 that the pseudo-skin factor produced by the inclined angle has a positive impact on the productivity of the gas reservoir. The greater the pseudo-skin factor, the greater the impact on productivity. The following figure shows the relationship between the inclined angle and the pseudo-skin factor, and the $\mathrm{Pwf}<\mathrm{P}<\mathrm{Pcr}$ region IPR curve calculated about the $\mathrm{H}$ gas reservoir X1 well at different angles. Figure 7 shows that the rate of the pseudo-skin factor increases with increasing angle ; Figure 8 shows that the daily gas production increases with the increasing inclined angle. The smaller the pressure and the larger the angle is, the more the daily gas production will be. It shows that, for inclined wells, the stimulation effect is more obvious when the inclined angle is over $60^{\circ}$.

Figure 9 shows that the stress sensitivity effect has a negative impact on the productivity of condensate gas reservoirs. When the stress sensitivity coefficient is larger, Zone II will be enlarged. Because of a steady-state model with fixing the outer boundary pressure of Zone I, when the stress-sensitive effect is considered in Zone I, the internal pressure reduces rapidly. The following figure shows a model, whose stress sensitivity coefficient is 0.01 . It can be seen that under the large stress sensitivity, when the bottom hole pressure is lower than the critical flow pressure, the outer boundary of the Zone II climbs rapidly with condensate flowing at the bottom of well. Thus, the productivity of gas wells are adversely affected. 


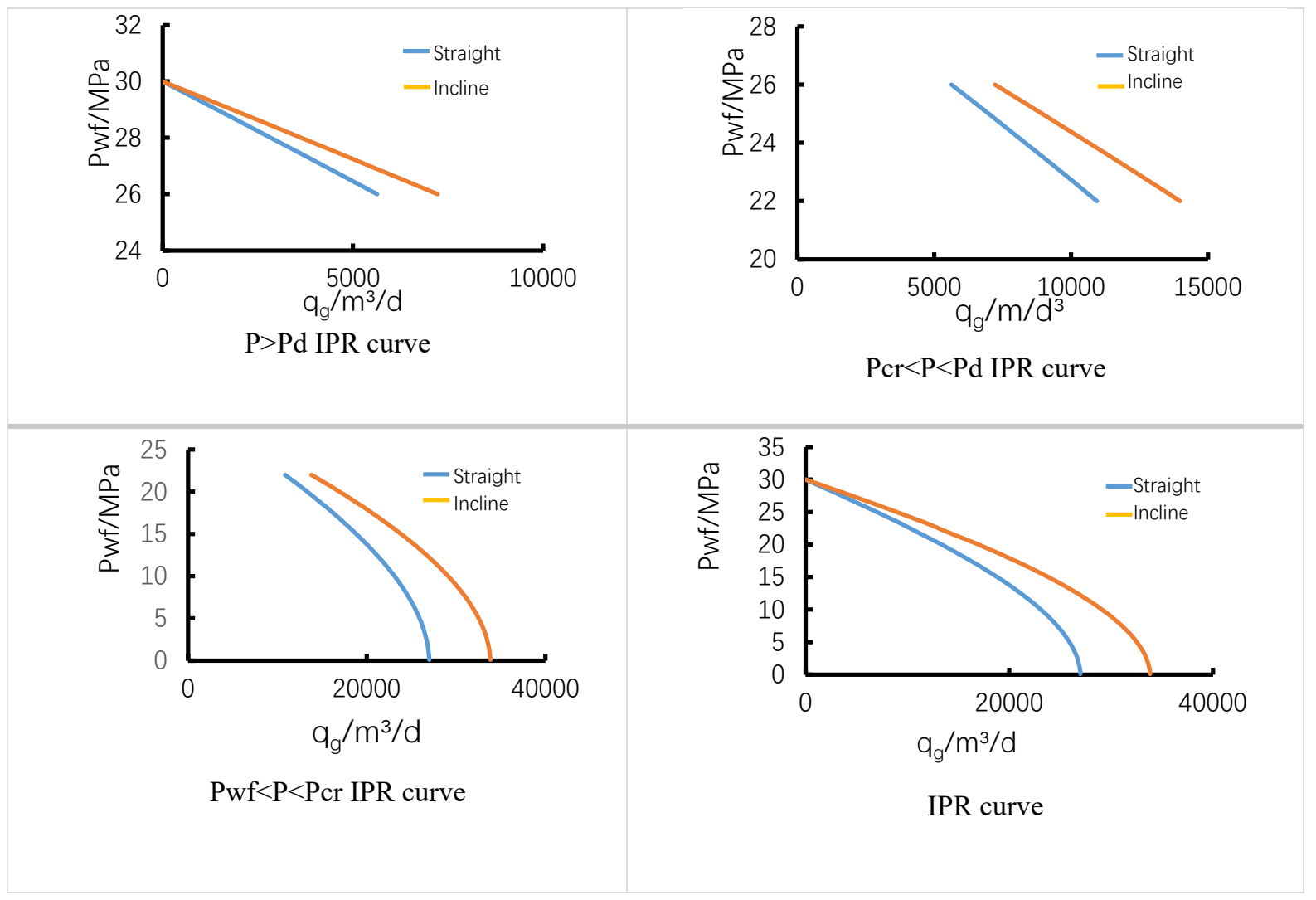

Figure 6. IPR curve

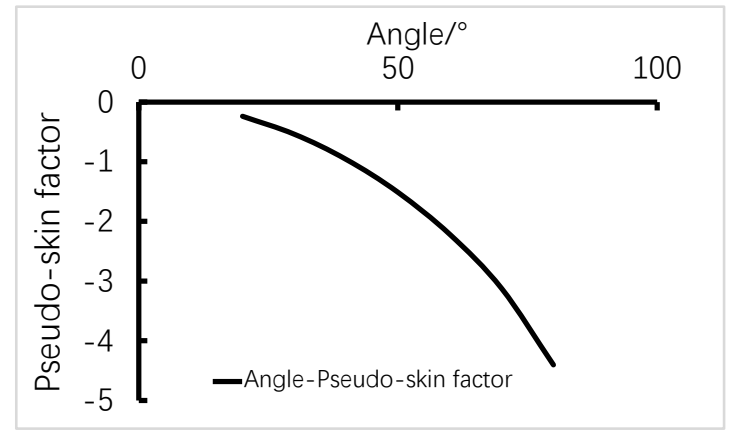

Figure 7. Angles- Pseudo-skin factor

The starting pressure gradient has a negative impact on the productivity of condensate gas reservoirs. But for the $\mathrm{H}$ condensate gas reservoir, the starting pressure gradient is only considered in Zone I. It can be seen from the figure 10 that the starting pressure gradient has a relatively small impact on productivity because of the small radius of Zone I. In this case, the maximum value is $18.1 \mathrm{~m}$. However, the starting pressure gradient influences the productivity, in Figure 10, which is related to the zone radius.

Figure 11 shows that HSND has a negative impact on the productivity of condensate gas reservoirs. Because the model used in this paper only considers it in Zone II, and

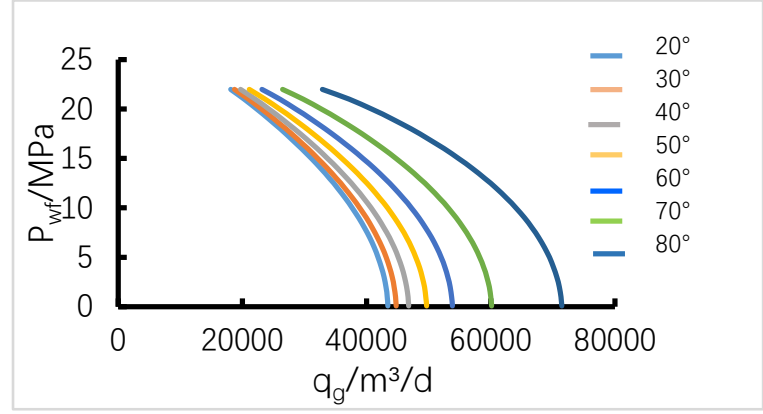

Figure 8. IPR curve at different tilt angles

the IPR curve with formation pressure in the $\mathrm{Pcr}<\mathrm{P}<\mathrm{Pd}$ stage is used to analyze the HSND. When the bottom hole pressure is $22 \mathrm{MPa}$, decline of the gas well production is caused by the HSND in the $\mathrm{H}$ gas reservoir X1, which has reached 1,200 cubic meters. With the declining of pressure, the productivity highly decreases. Therefore, for lowpermeability condensate gas reservoirs, the HSND causes larger losses. It can be seen from the figure that the lower the bottom hole pressure is, the more obvious the HSND will impact. The reason is the greater the pressure difference is, the greater the fluid velocity and the greater the turbulence effect will be. 


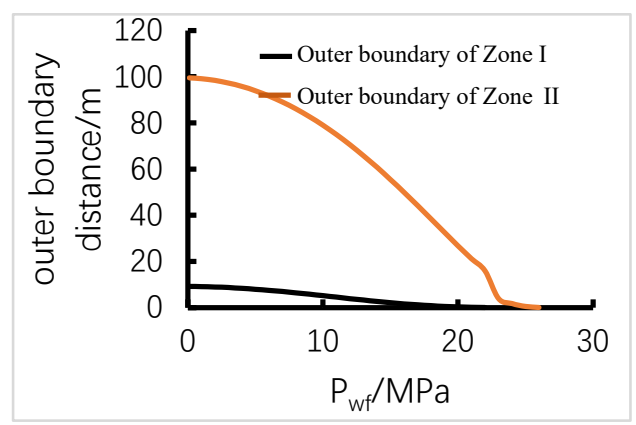

Figure 9. Stress sensitivity on zone radius

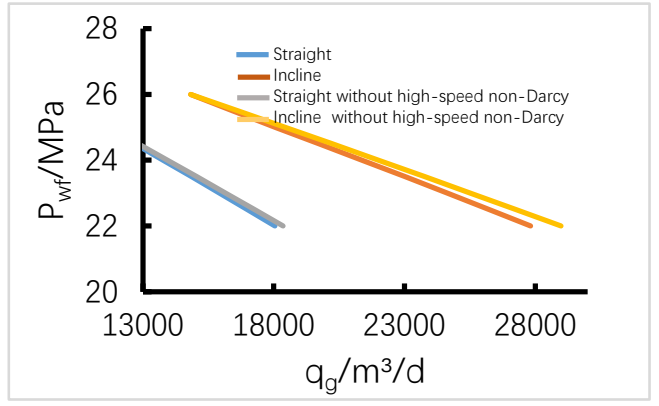

Figure 11. High-speed non-Darcy effect

It can be seen from Figure 12 that water production from gas wells has a negative impact on gas well productivity. For gas reservoirs, water production has the greatest impact on gas reservoirs, which can be known by analyzing the IPR curve in the $\mathrm{Pwf}<\mathrm{P}<\mathrm{Pcr}$ range. As the pressure difference increases, the greater the degree of productivity decline, the greater the impact of water production on gas well productivity.

It can also be seen from Figure 13 that the impact of water production on gas well productivity is more obvious than the well type. In the production of condensate gas reservoirs, we can increase the productivity by increasing the inclined angle. But if there is water invasion in the gas reservoir, water invasion control should be the first priority. At the same time, we can see that the impact of water production on the productivity of inclined wells is greater than vertical wells. That is, the higher the gas reservoir productivity is, the greater water intrusion will impact on productivity; under the high pressure difference, the impact of water invasion on productivity is much greater than low pressure difference. Indicating that, in order to improve the recovery rate of condensate gas reservoirs, it is necessary to control the pressure with water producing.

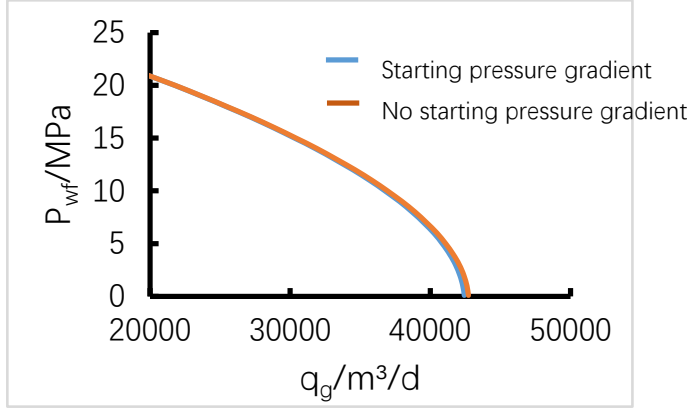

Figure 10. Starting pressure gradient on productivity

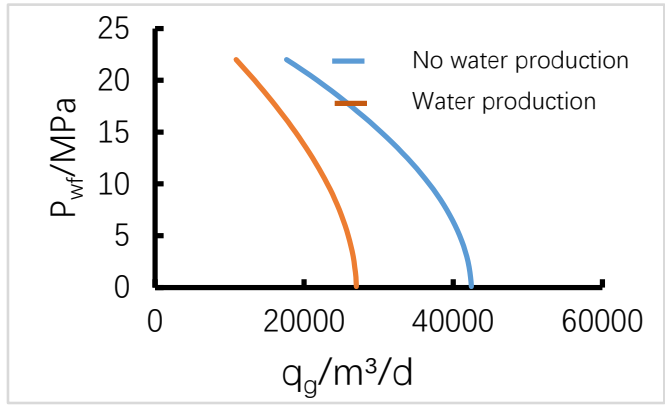

Figure 12. Water production on gas well productivity

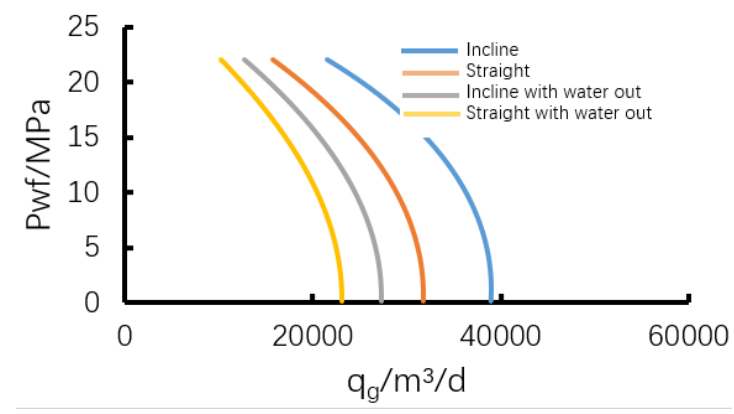

Figure 13 IPR curve under different conditions

\section{Conclusion}

(1) The problem of high gas-oil ratio is transformed into a gas-phase single-phase problem. Therefore, the derivation of the oil-gas-water three-phase productivity formula is converted into a gas-water two-phase problem. Due to the gas-water two-phase seepage characteristics, the influence of gas well water production is considered as the additional-skin factor, the influence of the inclined angle is considered as the pseudo-skin factor. Eventually, the gas phase productivity equation is derived. The method is simpler, the number of production required is relatively smaller, the accuracy is higher, and the practicability is stronger.

(2) Based on the different seepage characteristics of 
fluids under different formation pressures, the productivity equation under different bottom hole pressures is derived, which is closer to the actual.

(3) Combined with the case analysis of well $\mathrm{X} 1$ in $\mathrm{H}$ gas reservoir, the influence of inclined angle, water production, stress sensitivity, starting pressure gradient and HSND on gas well productivity is analyzed. Result in the greater the inclined angle, the greater the productivity. If it is over $60^{\circ}$, the gas well has an obvious stimulation effect; the water production has the greatest impact on the gas well, which is much higher than the gain in gas well productivity due to the inclined; stress sensitivity, starting pressure gradient and HSND has a negative impact.

\section{Acknowledgments}

Supported by the National Major Science and Technology Project "Research on percolation Mechanism and Development Technology Policy of Thick Heterogeneous Gas Reservoirs" (No. :2016ZX05027-004-005).

\section{References}

1. Fetkovich, M. J. (1973) The Isochronal Testing of Oil Wells. In: SPE. 4529.

2. Gringarten, C. Al-Lamki, A. Daungkaew, S. (2000) Well Test Analysis in Gas-Condensate Reservoirs. In: SPE. 62920

3. Lu, J L. Zhang, H. Chang, B H. Cao, W. \& Sun, H D. (2018) A new deliverability evaluation method of gas condensate wells in gas-liquid two-phase state. J. Natural Gas Industry B, $5: 583-588$

4. Mazloom J. Rashidi, F. (2006) Use of Two-Phase Pseudo Pressure Method to Calculate Condensate Bank Size and Well Deliverability in Gas Condensate Reservoirs. J. Petroleum Science and Technology, $24: 2,145-156$

5. Arlld. (2006) Two-Phase Pressure Test Analysis. In: SPE. 10224

6. Johnson, C. Jamiolahmady, M. (2018) Production data analysis in gas condensate reservoirs using type curves and the equivalent single phase approach. J. Journal of Petroleum Science and Engineering, 171.

7. He, Z X. Sun, L. Li, S L. (1996) A new method for productivity analysis of condensate gas wells. J. Natural Gas Industry, 16(05): 32-36

8. Xie, X L. Luo, K. Song, W J. (2001) Research on the new productivity equation of condensate gas. J. Acta Petrolei Sinica, 22(3):37-42

9. Huang, Q H. Li, S L. Gao, G H. etc. (2001) Condensate oil and gas seepage considering the effect of porous media adsorption. J. Natural Gas Industry, 21(2): $75-78$ 